\title{
ACONTECIMIENTOS VITALES ESTRESANTES, RESILIENCIA Y AJUSTE ADOLESCENTE
}

\author{
Alfredo Oliva, Jesús M. JimÉnez, Águeda Parra e InMaculada SÁnchez-QueiJA ${ }^{1}$ \\ Facultad de Psicología, Universidad de Sevilla \\ ${ }^{1}$ Facultad de Psicología, Universidad de Nacional de Educación a Distancia, Madrid
}

\begin{abstract}
Resumen: El presente estudio analizó el impacto de la ocurrencia de acontecimientos vitales estresantes en el ajuste adolescente. Con este objetivo, 101 adolescentes fueron estudiados en dos ocasiones, cuando se encontraban en la adolescencia media (15-16 años) y dos años después (17-18 años). Los participantes cumplimentaron instrumentos sobre acontecimientos vitales estresantes, relaciones familiares y ajuste adolescente. Los resultados mostraron la influencia significativa de este tipo de acontecimientos sobre la satisfacción vital, los problemas emocionales y, sobre todo, los problemas exteriorizantes. Además, la utilización de un análisis centrado en el sujeto reveló que los adolescentes resilientes (mucha adversidad y pocos problemas exteriorizantes) gozaban de relaciones familiares de mayor calidad que los adolescentes maladaptados (mucha adversidad y muchos problemas exteriorizantes). Por lo tanto, unas relaciones familiares positivas durante la adolescencia pueden ser consideradas como un factor de protección ante las consecuencias negativas de los acontecimientos vitales estresantes.
\end{abstract}

Palabras clave: problemas internalizantes, problemas externalizantes, resiliencia, adolescencia

\section{Stressful life events, resilience and adolescent adjustment}

\begin{abstract}
This study analyses the impact of the occurrence of stressful life events in adolescent adjustment. To this end 101 adolescents were studied twice, first when they were in middle adolescence (15-16 years) and then two years later (17-18 years). Participants filled out questionnaires about stressful life events, family relationships and adolescent adjustment. The results show a significant influence of this type of events on life-satisfaction, emotional problems and, specially, on externalizing problems. Besides, the use of a person-centered analysis revealed that resilient adolescents (high adversity and few externalizing problems) had family relationships of a better quality than maladaptive peers (high adversity, many externalizing problems). So, supportive family relationships during adolescence can be considered a protective factor against the negative consequences of stressful life events.
\end{abstract}

Keywords: internalizing problems, externalizing problems, resilience, adolescence.

\section{INTRODUCCIÓN}

La ocurrencia de acontecimientos vitales estresantes es uno de los factores de riesgo para el ajuste adolescente citados con más frecuen-

Recibido 3 mayo 2007; aceptado 2 noviembre 2007.

Este estudio fue financiado con la ayuda concedida a los autores por el Ministerio de Educación, Cultura y Deportes: BSO2022-03022

Correspondencia: Alfredo Oliva Delgado, Departamento de Psicología Evolutiva y de la Educación. Facultad de Psicología, c/ Camilo José Cela, s/n, 41018 Sevilla (España). Correo-e: oliva@us.es cia por los investigadores, existiendo una amplia evidencia empírica sobre el aumento de los problemas emocionales y depresivos en respuesta a la ocurrencia de algunos de estos acontecimientos (Aseltine, Gore y Colten, 1994; Ge, Lorenz, Conger, Elder y Simmons, 1994; Kim, Conger, Elder y Lorenz, 2003; Kraaij et al., 2003). De hecho, los cambios y tareas evolutivas que deben afrontarse durante la adolescencia convierten a esta etapa en un periodo complicado y difícil para los jóvenes, en el que la ocurrencia de algunos acontecimientos vitales puede suponer una dosis añadida de estrés que 
disminuya su satisfacción vital y aumente el riesgo de desarrollar problemas emocionales. Como ponen de manifiesto algunas investigaciones (Gest, Neeman, Hubbard, Masten y Tellegen, 1993; Jackson y Warren, 2000; Kim et al., 2003), el impacto de estos acontecimientos se extiende a los problemas de comportamiento, llegando incluso a relacionarse con el aumento de actividades antisociales. Aunque algunos autores han señalado el interés de investigar la relación entre acontecimientos estresantes específicos y consecuencias psicológicas (McMahon, Grant, Compas, Thurm y Ey, 2003), muchos de estos acontecimientos suelen estar relacionados entre sí y tienen un efecto acumulativo (Masten y Wright, 1998). Por ello, los inventarios que incluyen un listado de acontecimientos vitales y que proporcionan un índice global de adversidad suelen ser uno de los métodos más empleados por los investigadores. Típicamente, estos inventarios incluyen acontecimientos negativos representativos del tipo de experiencias estresantes más frecuentes durante la adolescencia (Kirchner y Forns, 2000).

El hecho de que no todos los sujetos se vean afectados de igual manera por los estresores vitales es expresión de la variabilidad natural de la respuesta individual que tiene que ver con determinados mecanismos de vulnerabilidad, pero que también puede explicarse en términos de resiliencia o resistencia ante la adversidad. Aunque algunos autores relacionan la resiliencia con el crecimiento postraumático (Manciaux, Cyrulnik y Vanistendael, 2003), en este trabajo se adoptará un enfoque más restrictivo, que la consideran como el resultado del proceso de afrontamiento que permite al individuo mantener su equilibrio y mostrar una buena adaptación a pesar de estar expuesto a una dosis de adversidad significativa (Becoña, 2006; Lemos, 2003; Luthar, 2006; Luthar, Cicchetti y Becker, 2000; Masten, 2001; Vera, Carabelo y Vecina, 2006).

En este sentido, el estudio de los factores que protegen a los adolescentes de las consecuencias negativas de los sucesos vitales es importante ya que nos ayuda a comprender los mecanismos mediante los cuales estos sucesos generan problemas adaptativos (Grant, Com- pas, Stuhlmacher, Thurm, McMahon y Halpert, 2003). Entre estos factores protectores destacan algunos de carácter contextual como el apoyo social o la presencia de padres o cuidadores competentes (Masten, 2001).

La investigación sobre resistencia ha empleado en los últimos años dos enfoques distintos pero complementarios: los estudios centrados en las variables y los centrados en el individuo (Becoña, 2006; Masten, 2001). En los primeros se emplean análisis estadísticos multivariantes para probar las relaciones entre variables de riesgo o adversidad, variables de competencia del sujeto, y cualidades del individuo o de su contexto que pudieran actuar como factores de protección. En cambio, los estudios que emplean un diseño centrado en el individuo proceden mediante la comparación de grupos de sujetos con diferente grado de ajuste y de exposición al riesgo, siendo de especial interés la comparación entre los sujetos resilientes y los que, por el contrario, han sucumbido ante la adversidad y muestran desajustes (Masten, Hubbard, Gest, Tellegen, Garmezy y Ramírez, 1999). Un hallazgo común de estos estudios es que los niños y adolescentes que resisten y los que muestran desajustes difieren entre sí, ya que los primeros gozan de un clima familiar más positivo y caracterizado por unos vínculos emocionales más estrechos. Por ello, unas buenas relaciones familiares son consideradas un importante factor de protección ante el impacto negativo de los estresores vitales (Elder y Conger, 2000; Luthar y Zelazo, 2003). Distintos autores (Bonano, 2004; Dumont y Provost, 1999) también han hecho referencia al papel protector de otras variables como las relaciones del adolescente con el grupo de iguales y determinadas características o rasgos del sujeto, como las estrategias de afrontamiento de problemas o la autoestima.

La adaptación o el ajuste de los adolescentes resilientes ha sido definido de maneras muy diferentes en términos de buena salud mental, satisfacción vital, capacidad funcional y competencia social, aunque la ausencia de problemas conductuales es uno de los indicadores más utilizados (Luthar et al., 2000). Sin embargo, algunos autores como Luthar (1991) han señalado la importancia de observar también 
otros índices, ya que algunos estudios han encontrado en jóvenes resilientes un elevado nivel de malestar emocional que contrasta con su ajuste conductual.

El presente estudio se llevó a cabo con el objetivo de analizar las consecuencias para el ajuste adolescente, tanto interno como externo, de los acontecimientos vitales estresantes. Para ello, se construyó una escala autocumplimentable que incluía 29 sucesos adversos frecuentes durante la adolescencia. En primer lugar, y siguiendo un enfoque centrado en las variables, procedimos a analizar la relación entre el índice acumulado de estresores experimentados en los últimos años por los adolescentes de la muestra y su ajuste psicológico definido como la ausencia de problemas interiorizantes y exteriorizantes y una elevada satisfacción vital. En segundo lugar, mediante un enfoque centrado en el sujeto, tratamos de detectar factores de protección referidos a las relaciones familiares. Para ello, y siguiendo la propuesta de Masten et al., (1999), procedimos a formar 4 grupos de sujetos (resilientes, adaptados, vulnerables y maladaptados) sobre la base de la ocurrencia de acontecimientos vitales estresantes (AVE) y la mayor o menor presencia de problemas de exteriorizantes o de conducta.

El carácter longitudinal del estudio nos permitió además analizar las trayectorias que seguían entre la adolescencia media y tardía las variables de estudio en cada uno de esos 4 grupos, así como la posible influencia de la ocurrencia de acontecimientos estresantes sobre el aumento de los problemas emocionales y conductuales entre la adolescencia media y la tardía.

Las hipótesis planteadas en el estudio fueron las siguientes:

La ocurrencia de AVE estará relacionada con la presencia de problemas interiorizantes y exteriorizantes y con una menor satisfacción vital.

Los adolescentes resilientes (muchos AVE y buen ajuste exteriorizado) tendrán relaciones familiares caracterizadas por una alta adaptabilidad y cohesión emocional, sobre todo en comparación con los maladaptados (muchos AVE y mal ajuste exteriorizado).

Los adolescentes resilientes, a pesar de su ajuste comportamental, mostrarán más proble- mas emocionales y menos satisfacción vital que los sujetos que hayan experimentado pocos AVE (vulnerables y adaptados).

\section{MÉTODO}

\section{Muestra}

Este trabajo presenta parte del seguimiento longitudinal de un grupo de chicos y chicas a lo largo de su adolescencia. El punto de partida es una investigación previa transversal en la que la muestra estuvo compuesta por 513 adolescentes de edades comprendidas entre los 12 y los 19 años y pertenecientes a 10 centros educativos diferentes de Sevilla y su provincia. La elección de los colegios e institutos donde se seleccionó a los adolescentes se llevó a cabo teniendo en cuenta criterios representativos como su pertenencia al mundo rural o urbano, su titularidad - pública o privada concertada- $\mathrm{y}$ el nivel socioeconómico de las familias.

La segunda fase de la investigación consistió en el seguimiento de los chicos y chicas que en el estudio anterior se encontraban en la adolescencia inicial, entre los 12 y los 14 años $(M=13,11$ y $D T=0,44)$. Este seguimiento se realizó durante más de cinco años, hasta que cumplieron los 18 ó 19 años. Así, estos jóvenes completaron los instrumentos de evaluación en su adolescencia inicial, media y tardía. La muestra final estuvo compuesta por 101 adolescentes, 38 chicos y 63 chicas. Las edades medias en la adolescencia media y tardía fueron $15,38(D T=0,56)$, y $17,85(D T=0,52)$, respectivamente.

El trabajo que se presenta aquí se refiere a los datos correspondientes a las fases 2 y 3 de este estudio longitudinal, es decir los referidos a la adolescencia media y tardía, que serán denominados en este artículo Tiempo 1 (T1) y Tiempo 2 (T2).

Para identificar las posibles diferencias entre los jóvenes que continuaron en la investigación y aquellos que no lo hicieron, realizamos el Análisis de casos perdidos. Nuestros resultados indican que entre los sujetos que continuaron en la investigación hay más chicas que chicos, $\chi^{2}=4,05, p<0,05$, y menos hijos de padres de 
nivel educativo bajo, $\chi^{2}=6,52, p<0,05$. No obstante, son semejantes en cuanto a su hábitat -rural vs. urbano- y al tipo de centro educativo al que asisten -público vs. privado-. Tampoco aparecieron diferencias significativas entre unos y otros en las variables referidas a las relaciones familiares y al ajuste adolescente.

\section{Instrumentos}

Los instrumentos utilizados en el estudio fueron:

Family Adaptability and Cohesion Scale (FACES II) (Olson, Portner y Lavee, 1985). Se trata de una escala desarrollada para evaluar las relaciones familiares según la percepción de padres o adolescentes. Compuesto por 30 ítems que permiten evaluar en las relaciones familiares la cohesión emocional (T1: $M=56,86$; $D T=9,82 ;$ rango $=24-77 ; \alpha$ de Cronbach $=0,84$ / T2: $M=55,61 ; D T=10,09 ;$ rango $=29$ - 79; $\alpha=0,87)$ y la adaptabilidad (T1: $M=$ 49,$22 ; D T=7,03 ;$ rango $=23-65 ; \alpha=0,74$ / T2: $M=47,40 ; D T=7,80 ;$ rango $=26-65$; $\alpha=0,81)$.

Youth Self-Report (Achenbach, 1991). Escala autocumplimentable compuesta por 113 ítems y diseñada para su uso con adolescentes entre los 12 a los 18 años de edad. Analiza la presencia de problemas emocionales o interiorizantes $(\mathrm{T} 1: M=13,37 ; D T=8,79$; rango $=0$ $-46 ; \alpha=0,89$ / T2: $M=12,29 ; D T=7,51$; rango $=1-38 ; \alpha=0,86)$ y comportamentales o exteriorizantes (T1: $M=13,17 ; D T=6,72$; rango $=1-38 ; \alpha=0,76 / \mathrm{T} 2: M=13,60 ; D T=$ $6,21 ;$ rango $=1-31 ; \alpha=0,77)$.

Escala de Satisfacción vital. Se trata de una adaptación del instrumento de Huebner (1991) Students' Life Satisfaction Scale (SLSS). Fueron seleccionados 5 ítems que evalúan la satisfacción de chicos y chicas con diferentes aspectos de sus vidas (T1: $M=$ 17,$89 ; D T=4,49 ;$ rango $=5-25 ; \alpha=0,83$ / T2: $M=17,94 ; D T=4,03 ;$ rango $=6-25$; $\alpha=0,77)$.

Acontecimientos Vitales Estresantes (AVE). Inventario autocumplimentable diseñado para este estudio y consistente en un listado de 29 sucesos negativos relativos a sí mismo y a otras personas significativas, que pueden ser experimentados por el adolescente en los contextos de familia, escuela o grupo de iguales (divorcio o separación de los padres, muerte de un familiar, repetición de curso, ruptura con la pareja, etc.). Estos acontecimientos fueron seleccionados a partir de una revisión bibliográfica y un posterior estudio piloto. Los participantes debían indicar si algunos de estos sucesos habían ocurridos durante los últimos 3 años. Cada ítem recibía una puntuación de 1 si había ocurrido o de 0 si no había tenido lugar. La puntuación total se obtenía mediante la suma de los 29 items $(M=6,28$; $D T=3,17$; rango $=0-15)$. Además, los adolescentes señalaban en una escala de 1 a 10 , el impacto emocional que cada uno de los sucesos ocurridos había tenido en ellos. Se obtenía una puntuación total sobre el impacto emocional mediante la suma de los ítems individuales $(M=32,84 ; D T=22,20 ;$ rango $=0-114)$.

\section{Procedimiento}

El primer paso fue seleccionar los centros educativos y contactar con su equipo directivo para explicarles la investigación y solicitar su colaboración. Una vez que aceptaron participar, seleccionamos las aulas en las que recogeríamos los datos. A continuación enviamos una carta a los padres y madres solicitando el permiso para que sus hijos colaboraran en la investigación. En la segunda y tercera recogida de datos, que son las utilizadas en este artículo, algunos adolescentes no estaban escolarizados o lo estaban en centros diferentes, por lo que tras contactar con ellos, y una vez que aceptaron colaborar, cumplimentaron un cuestionario de forma individual en el centro educativo en el que estaban o habían estado escolarizados.

\section{RESULTADOS}

Los acontecimientos vitales estresantes que experimentaron más adolescentes fueron el traslado de clase $(72,3 \%)$, la muerte de un 
familiar cercano $(54,5 \%)$ y la ruptura de la relación con la pareja $(45,5 \%)$. En el lado contrario, el acontecimiento menos frecuente fue el embarazo de la joven o de la pareja del adolescente $(0 \%)$. A continuación aparecen tres sucesos con idéntica frecuencia (4\%) pero con muy diferente impacto, como sufrir acoso o abuso sexual, falta de interés de los padres y un nuevo emparejamiento de los padres. En cuanto al impacto emocional de los acontecimientos, el que más negativamente afectó a los adolescentes de nuestro estudio fue el fallecimiento de un familiar ( 8,1 sobre un máximo de 10), seguido por las discusiones y la ruptura con el mejor amigo $(7,9)$, la enfermedad de un familiar $(7,9)$, el engaño o la traición de la pareja $(7,9)$ y haber sufrido acoso o abuso sexual $(7,7)$. Si combinamos la frecuencia y el impacto de los sucesos vitales, parece claro que el suceso que más afecta a los adolescentes es la muerte de un familiar, ya que es un acontecimiento que ha experimentado más de la mitad de los adolescentes y presenta el impacto emocional más elevado. Con alto impacto y considerable frecuencia destacan la ruptura de la relación de pareja, las broncas y conflictos con los padres y la ruptura con el mejor amigo. Entre los sucesos con baja frecuencia y alto impacto destaca haber sufrido acoso o abuso sexual y que algún familiar cercano padezca alguna minusvalía o enfermedad mental grave. Nuestros datos revelaron que en términos generales no existen diferencias significativas entre chicos y chicas en la ocurrencia o el impacto emocional negativo de los estresores.

Para analizar la relación entre los AVE y el ajuste adolescente que habíamos planteado en la $1^{\text {a }}$ hipótesis, se procedió en primer lugar al análisis de correlaciones. Como puede observarse en la Tabla 1, la ocurrencia de AVE se asoció a la presencia de problemas emocionales y de conducta, y a una menor satisfacción vital. En lo referente al impacto emocional negativo de los acontecimientos, también se observaron correlaciones significativas con las dos dimensiones de ajuste del YSR y, de nuevo negativamente, con satisfacción vital.

Para profundizar en estas relaciones, decidimos llevar a cabo varios análisis de regresión múltiple usando como variables dependientes la satisfacción vital, los problemas interiorizantes y los problemas exteriorizantes, todas ellas correspondientes a Tiempo 2. Como variables predictoras se incluyeron los AVE y las mismas variables anteriores pero con los valores correspondientes a Tiempo 1, en lo que se denomina un modelo auto-regresivo (Stollmiller y Bank, 1995). Este tipo de análisis nos permite determinar qué variables explican la varianza de la variable dependiente que no es explicada por los valores de esa misma variable en un momento anterior, o lo que es lo mismo, si el cambio que se produce en cada una de estas variables entre T1 y T2 puede atribuirse a la ocurrencia de acontecimientos estresantes. Los resultados de estos modelos indicaron que los AVE influyeron significativamente en los problemas exteriorizantes y en la satisfacción vital, aunque no en los problemas interiorizantes. Así, como puede verse en las Tablas 2 y 3 , nuestros datos indican que la ocurrencia de AVE contribuyó de forma significativa al aumento de los problemas comportamentales y a la disminución de la satisfacción vital entre T1 y T2.

Tabla 1. Correlaciones entre acontecimientos vitales estresantes y ajuste adolescente en Tiempo 1 y Tiempo 2

\begin{tabular}{lcccccc}
\hline & \multicolumn{2}{c}{$\begin{array}{c}\text { Problemas } \\
\text { exteriorizantes }\end{array}$} & \multicolumn{2}{c}{$\begin{array}{c}\text { Problemas } \\
\text { interiorizantes }\end{array}$} & \multicolumn{2}{c}{ Satisfacción vital } \\
& $\mathrm{T} 1$ & $\mathrm{~T} 2$ & $\mathrm{~T} 1$ & $\mathrm{~T} 2$ & $\mathrm{~T} 1$ & $\mathrm{~T} 2$ \\
\hline Ocurrencia AVE & $0,29 * *$ & $0,42^{* * *}$ & $0,26 * *$ & $0,29 * *$ & $-0,20^{*}$ & $0,37 * * *$ \\
Impacto emocional AVE & $0,28^{* *}$ & $0,30^{* *}$ & 0,18 & $0,38^{* * *}$ & $-0,21^{*}$ & $0,36^{* * *}$ \\
\hline
\end{tabular}

$* * * p<0,001, * * p<0,01 * p<0,05$ 
Tabla 2. Modelo autoregresivo prediciendo problemas exteriorizantes en Tiempo 2

\begin{tabular}{llcc}
\hline Variables predictoras & Beta & $\mathrm{t}$ & $\mathrm{R}^{2}$ \\
\hline Sucesos vitales & $0,25^{* *}$ & 3,39 & 0,50 \\
Problemas exteriorizantes T1 & $0,59^{* * *}$ & 7,84 & \\
\hline
\end{tabular}

$* * * p<0,001, * * p<0,01 * p<0,05$

Para comprobar si, como habíamos expuesto en la segunda hipótesis, los adolescentes resilientes tenían mejores relaciones familiares que quienes presentaban peor adaptación en presencia de AVE, y utilizando esta vez un enfoque centrado en el sujeto, procedimos, en primer lugar, a la formación de los cuatro grupos de adolescentes en función de la mayor o menor adversidad experimentada y de la mayor o menor frecuencia de problemas exteriorizantes. Combinando estas 2 dimensiones, se obtuvo un primer grupo de sujetos resilientes $(n=13)$ caracterizados por experimentar un número de AVE por encima de la mediana y presentar una puntuación en problemas de conducta por debajo de la mediana. El segundo grupo $(n=39)$, que denominamos adaptados, lo forman sujetos con puntuaciones inferiores a la mediana en esas dos variables. El tercer grupo estaba compuesto por los sujetos vulnerables $(\mathrm{n}=18)$, es decir, adolescentes que presentaban muchos problemas de conducta aunque habían experimentado pocos AVE. Por su parte, el último grupo reunía a adolescentes que llamamos maladaptados $(\mathrm{n}=31)$, y que se caracterizaban por mostrar puntuaciones superiores a la mediana en ambas variables. El reducido tamaño de nuestra muestra nos llevó a usar un criterio poco restrictivo - puntuaciones por encima o por debajo de la mediana- para definir tanto el riesgo como la competencia conductual. Formados los grupos, no se observó una relación significativa entre el sexo y pertenencia a una $\mathrm{u}$ otra de estas 4 agrupaciones.
A partir de aquí, procedimos, en primer lugar, a la comparación de estos cuatro grupos para ver si los sujetos resilientes tenían algunas características familiares que los diferenciaban de aquellos que no mostraron la misma adaptación en presencia de adversidad. En segundo lugar, tratamos de comprobar si esos adolescentes resilientes, a pesar de su ajuste comportamental, mostraban problemas interiorizantes o emocionales.

Como puede verse en la Tabla 4, el análisis de varianza puso de manifiesto la existencia de diferencias significativas en las relaciones familiares de los cuatro grupos, tanto en Tiempo 1 como en Tiempo 2. La excepción a esta tendencia fue la dimensión de adaptabilidad en Tiempo 2, donde no se aprecian diferencias significativas. Cuando analizamos los resultados en $\mathrm{T} 1$, se observó, en primer lugar, que el grupo de adolescentes resilientes puntuó más alto que el resto de los grupos en adaptabilidad, $F(3,97)$ $=4,34, p<0,01$, y cohesión, $F(3,97)=8,93$, $p<0,01$. En el otro extremo, destacaron los sujetos maladaptados, cuyas puntuaciones fueron significativamente más bajas en estas dos mismas dimensiones. Para afinar aún más en el estudio de las diferencias entre los grupos se procedió al análisis post hoc mediante la prueba Tukey, que puso de manifiesto que el grupo de adolescentes resilientes se distinguía significativamente en estas dimensiones del grupo de los adolescentes vulnerables (adaptabilidad, $p<$ $0,05$; cohesión, $p<0,01)$ y del de maladaptados (adaptabilidad, $p<0,05$; cohesión, $p<0,01$ ).

Tabla 3. Modelo autoregresivo prediciendo satisfacción vital en Tiempo 2

\begin{tabular}{lcrc}
\hline Variables predictoras & Beta & $\mathrm{t}$ & $\mathrm{R}^{2}$ \\
\hline Sucesos vitales & $-0,28^{* *}$ & $-3,36$ & 0,37 \\
Satisfacción vital T1 & $0,49^{* * *}$ & 5,95 & \\
\hline
\end{tabular}

${ }^{* * *} p<0,001, * * p<0,01,{ }^{*} p<0,05$ 
Tabla 4. Puntuación media de los cuatro grupos de sujetos en variables familiares, problemas interiorizantes y satisfacción vital en los dos momentos de la recogida de datos

\begin{tabular}{|c|c|c|c|c|c|c|c|c|}
\hline & \multicolumn{4}{|c|}{ TIEMPO 1} & \multicolumn{4}{|c|}{ TIEMPO 2} \\
\hline & $\begin{array}{l}\text { Adapta- } \\
\text { bilidad }\end{array}$ & Cohesión & $\begin{array}{l}\text { Probl. } \\
\text { Interior. }\end{array}$ & $\begin{array}{c}\text { Satisfac. } \\
\text { Vital }\end{array}$ & $\begin{array}{l}\text { Adapta- } \\
\text { bilidad }\end{array}$ & Cohesión & $\begin{array}{l}\text { Probl. } \\
\text { Interior. }\end{array}$ & $\begin{array}{c}\text { Satisfac. } \\
\text { Vital }\end{array}$ \\
\hline Resilientes & 53,23 & 63,92 & 13,23 & 19,84 & 47,76 & 58,84 & 10,92 & 19.07 \\
\hline Adaptados & 50,79 & 60,17 & 9,87 & 19,10 & 49.07 & 59,25 & 9.05 & 19,92 \\
\hline Vulnerables & 46,16 & 53.00 & 14,33 & 17,88 & 48,11 & 56,88 & 12,94 & 18.05 \\
\hline Maladaptados & 47,32 & 51,96 & 17,25 & 15,54 & 44,70 & 48,93 & 16,54 & 14,90 \\
\hline$p$ & 0,006 & 0,000 & 0,005 & 0,002 & 0,128 & 0,000 & 0,000 & 0,000 \\
\hline
\end{tabular}

También en Tiempo 2 aparecieron diferencias significativas entre los grupos, con la excepción ya comentada de la dimensión de adaptabilidad de FACES. Sin embargo, en la dimensión de cohesión estas diferencias sí fueron significativas, $F(3,97)=8,22, p<0,001$, revelando el análisis post hoc que el grupo de adolescentes maladaptados presentó puntuaciones más bajas que el resto de los grupos (adaptados, $p<0,001$; resilientes, $p<0,01$; vulnerables, $p<0,05$ ).

Finalmente, la hipótesis 3 planteaba las posibles dificultades emocionales de los sujetos resilientes, a pesar de su ajuste comportamental. En T1, la comparación entre los 4 grupos (Tabla 4) reveló diferencias significativas en las puntuaciones en la escala de problemas interiorizantes $F(3,97)=4,60, p<0,01$, aunque el análisis post hoc efectuado reveló que las diferencias sólo eran significativas cuando se comparaban los grupos de adolescentes adaptados y maladaptados $(p<0,01)$.El último de los aspectos estudiados en T1 fue la satisfacción vital, donde volvieron a aparecer diferencias significativas entre los grupos, $F(3,97)=$ $0,14, p<0,01$. El análisis post hoc determinó en este caso que las diferencias sólo eran significativas entre los adolescentes maladaptados, que presentaban la menor satisfacción vital, y los adaptados $(p<0,01)$ y los resilientes, $(p<$ $0,05)$ que se situaban claramente por encima.

En lo que se refiere a los problemas interiorizantes en T2, nuevamente se observaron diferencias, $F(3,97)=6,99, p<0,001$, aunque en este caso el análisis post hoc puso de manifiesto que las diferencias sólo eran significativas entre los adolescentes adaptados, que eran los más ajustados, y los maladaptados $(p<0,001)$.
En lo que se refiere a la satisfacción vital en T2, nuevamente aparecieron diferencias significativas, siendo los adolescentes maladaptados quienes presentaron menos satisfacción vital, $F(3,97)=12,59, p<0,001$. El análisis post hoc realizado mostró que el grupo de adolescentes maladaptados se diferenciaba significativamente del resto de los grupos (adaptados, $p<0,001$; resilientes, $p<0,01$; vulnerables, $p<0,05$ ).

Dado el carácter longitudinal del estudio, también analizamos los cambios entre T1 y T2 en cada uno de los cuatro grupos y en las diferentes variables estudiadas. El análisis de varianza de medidas repetidas nos indicó que en el grupo de sujetos resilientes se observó una disminución significativa entre T1 y T2 en las variables adaptabilidad, Traza de Pillai $F(1,99)=5,66, p<0,05$, y cohesión, Traza de Pillai $F(1,99)=5,40, p<0,05$. Por su parte, en el grupo de adolescentes maladaptados sólo resultó significativa la disminución en cohesión, Traza de Pillai $F(1,99)=4,29, p<0,05$. En el resto de grupos y variables no se observaron cambios significativos entre T1 y T2.

\section{DISCUSIÓN}

Los resultados de este estudio indicaron de forma clara que, tal como habíamos planteado en la primera hipótesis, los acontecimientos vitales estresantes son un importante factor de riesgo para el ajuste adolescente tanto interno como externo. Sin embargo, nuestros datos también ponen de manifiesto que no todos los sujetos de la muestra se vieron afectados de la misma manera. En este aspecto, parece claro 
que las relaciones familiares positivas, caracterizadas por la cohesión emocional y la adaptabilidad, actuaron para algunos sujetos como un factor protector de su ajuste comportamental en presencia de una dosis significativa de adversidad. Aunque otros estudios han encontrado evidencia empírica acerca de la influencia de este tipo de estresores sobre los problemas emocionales (Aseltine et al. 1994; Kim et al, 2003; Kraaij et al., 2003), nuestros datos parecen mostrar, sin embargo, una mayor relación con los problemas exteriorizantes. De hecho, el diseño longitudinal empleado nos permitió demostrar cómo la ocurrencia de estresores contribuyó al aumento de los problemas externalizantes, aunque no de los interiorizantes, entre Tiempo $1 \mathrm{y}$ Tiempo 2. Además, nuestros datos muestran que los AVE también influyeron en la disminución de la satisfacción vital entre estos dos momentos. Por lo tanto, podemos decir que los acontecimientos estresantes son un claro elemento perturbador del ajuste adolescente, y que algunos de estos acontecimientos, como la muerte de algún familiar, tienen un importante impacto emocional, lo que coincide con otros estudios llevados a cabo en nuestro país (Kirchner y Forns, 2000). Tal como se ha expuesto en otro lugar (Parra y Oliva, 2006), quizás sea la mayor estabilidad relativa durante la adolescencia de los problemas interiorizantes frente a la satisfacción vital o a los problemas comportamentales, que suelen fluctuar más, la responsable de que no se haya detectado la influencia de los AVE en el aumento de los problemas emocionales, aunque sí sobre sus valores absolutos al final de la adolescencia. El modelo auto-regresivo empleado en este estudio es poco sensible a la detección de influencias sobre el cambio en la variable dependiente cuando ésta es muy estable y, por lo tanto, la mayor parte de su varianza es explicada por esa misma variable medida en un momento anterior, como ocurre con los problemas interiorizantes.

En cuanto a los factores que protegen a los adolescentes de las consecuencias negativas de estos acontecimientos vitales, el enfoque tipológico empleado permitió comprobar que, tal como se había hipotetizado, tanto la adaptabilidad como la cohesión emocional eran más elevadas en las familias de los sujetos resilien- tes que en las de los sujetos maladaptados. De hecho, el grupo de sujetos resilientes era el que mostraba en la adolescencia media (T1) las relaciones familiares caracterizadas por la mayor cohesión y adaptabilidad. En la adolescencia tardía (T2), sin embargo, las diferencias no fueron tan favorables a los sujetos resilientes, aunque seguían puntuando mejor que el grupo de los maladaptados en estas dos dimensiones. Parece, por lo tanto, más que probable que estas positivas relaciones familiares en T1 hayan actuado protegiendo a algunos sujetos de las consecuencias negativas de los sucesos vitales experimentados, algo que ya ha sido sugerido por algunos autores (Luthar y Zelazo, 2003).

Sin embargo, la calidad de las relaciones familiares de los sujetos resilientes se habría visto afectada por la ocurrencia de AVE -téngase en cuenta que algunos de estos estresores se referían al contexto familiar-, y habría experimentado un ligero deterioro de T1 a T2, que el análisis de medidas repetidas reveló significativo. En contraste con lo anterior, en los grupos de sujetos expuestos a un nivel menor de adversidad (adaptados y vulnerables) no se observó ningún deterioro en las variables referidas a las relaciones familiares. Por su parte, en el grupo de los adolescentes maladaptados este descenso sólo fue significativo en cohesión emocional. En este último grupo, los niveles de la variable adaptabilidad ya eran muy bajos en Tiempo 1, por lo que no cabía esperar un descenso acusado a pesar de la ocurrencia de muchos AVE, mientras que en el caso de los adolescentes resilientes se partía de niveles bastantes más altos $\mathrm{y}$, por tanto, más susceptibles de empeoramiento como consecuencia de la ocurrencia de estresores, que como ya se ha comentado, en muchos casos afectaban al contexto familiar.

En términos generales, podemos decir que los resultados de este estudio están en consonancia con las investigaciones sobre riesgo y resistencia donde se propone que las relaciones familiares son uno de los principales factores que protegen a niños y niñas de los efectos adversos de los acontecimientos estresantes (Jackson y Warren, 2000 Quamma y Greenberg, 2004; Luthar, 2006). Y de la misma manera que los protegen durante la infancia también 
parecen protegerlos durante la adolescencia media y tardía, a pesar de que sobre esta etapa exista menos evidencia empírica (Maggs, Frome, Eccles y Barber, 1997; Masten et al., 1999; Muñoz-Rivas y Graña, 2001). Ello otorga un mayor interés a estos resultados, pues indican que, a pesar de la influencia creciente del grupo de iguales, la familia continúa teniendo un importante efecto protector sobre el ajuste conductual durante la adolescencia.

Los hallazgos de esta investigación longitudinal tienen desde nuestro punto de vista unas relevantes implicaciones prácticas, ya que nos sugieren la importancia de proporcionar apoyo a los padres de adolescentes, utilizando recursos como los programas de educación de padres, con el objetivo de que puedan establecer relaciones más comunicativas y afectuosas con sus hijos e hijas durante la adolescencia, ya que durante estos años es frecuente que en muchas familias aumenten los conflictos y disminuya la calidad de las relaciones (Oliva, 2006). En este contexto familiar más favorable, chicos y chicas se mostrarían más resistentes a las consecuencias negativas de muchos de los estresores propios de esta etapa del desarrollo.

En cuanto a la tercera hipótesis, referida a la posibilidad de un peor ajuste emocional de los sujetos resilientes, hay que señalar que no se vio confirmada por nuestros resultados, que coinciden con los de Masten et al. (1999) aunque no con los de Luthar (1991). Parece claro que en nuestro estudio no puede sustentarse la hipótesis referida a los problemas emocionales de los sujetos resilientes, ya que obtuvieron puntuaciones cercanas a las de los adaptados, especialmente en Tiempo 2.

Además, los adolescentes resilientes mostraron, tanto en Tiempo 1 como en Tiempo 2, una alta satisfacción vital y menos problemas interiorizantes que los que presentaban los adolescentes vulnerables y maladaptados. Podemos decir, por tanto, que el ajuste mostrado por los sujetos resilientes de nuestro estudio parece ir más allá del área comportamental, extendiéndose también a la esfera emocional.

No querríamos terminar sin referirnos a algunas limitaciones del estudio. En primer lugar, hay que señalar el pequeño tamaño mues- tral, que ha hecho que alguno de los grupos de adolescentes esté compuesto por un número muy reducido de sujetos. No obstante, el carácter longitudinal del estudio justifica y compensa en gran parte esta limitación. También tenemos que hacer referencia al hecho de que haber utilizado una única fuente de información, el propio adolescente, puede haber causado algún tipo de sesgo, incrementando la asociación entre las variables estudiadas. A pesar de todo, creemos que este estudio aporta datos de mucho interés sobre la importancia de las relaciones entre padres y adolescentes como factor de protección ante los efectos adversos de los acontecimientos vitales estresantes.

\section{REFERENCIAS}

Achenbach, T.M. (1991). Manual for the Child Behavior Checklist/4-18 and 1991 Profile. Burlington, VT: University of Vermont Department of Psychiatry.

Aseltine, R.F., Gore, S., y Colten, M.E. (1994). Depression and the social developmental context of adolescence. Journal of Personality and Social Psychology, 67, 252-264.

Becoña, E. (2006). Resiliencia: definición, caraterísticas y utilidad del concepto. Revista de Psicopatología y Psicología Clínica, 11, 125-146.

Bonano, G.A. (2004). Loss, Trauma, and Human resilience: How we underestimated the human capacity to thrive after extremely aversive events? American Psychologist, 59, 20-28.

Dumont, M., y Provost, M. (1999). Resilience in adolescence: Protective role of social support, coping strategies, self-esteem, and social activities on experience of stress and depression. Journal of Youth and Adolescence, 28, 343-363.

Elder, G.H., Jr., y Conger, R.D. (2000). Children of the land: Adversity and success in rural America. Chicago: University of Chicago Press.

Ge, X., Lorenz, F.O., Conger, R.D., Elder, G.H. Jr., y Simmons, R.L. (1994). Trajectories of stressful life events and depressive symptoms during adolescence. Developmental Psychology, 30, 467-483.

Gest, S.D., Neemann, J., Hubbard, J., Masten, A.S., y Tellegen, A. (1993). Parenting quality, adversity and conduct problems in adolescence: Testing process-oriented models of resilience. Development and Psychopathology, 5, 663-682.

Grant, K.E., Compas, B.E., Stuhlmacher, A., Thurm, A.E., McMahon, S., y Halpert, J. (2003). Stressors and child/ adolescent psychopathology: Moving from markers to 
mechanisms of risk. Psychological Bulletin, 129, 447-466. Huebner, E.S. (1991). Further validation of the Students' Life Satisfaction Scale: The independence of satisfaction and affect ratings. Journal of Psychoeducational Assessment, 9, 363-368.

Jackson, Y., y Warren, J. (2000). Appraisal, social support, and life events: Predicting outcome behavior in schoolage children. Child Development, 71, 1441-1457.

Kim, K.J., Conger, R.D., Elder, Jr., G.H., y Lorenz, F.O. (2003). Reciprocal influences between stressful life events and adolescent internalizing and externalizing problems. Child Development, 74, 127-143.

Kirchner, T., y Forns, M. (2000). Autopercepción de life events como factores de riesgo y de protección en adolescentes. Estudio diferencial. Revista de Psiquiatría de la Facultad de Medicina de Barcelona, 27, 63-76.

Kraaij, V., Garnefski, N., de Wilde, E.J., Dijkstra, A., Gebhardt, W., Maes, S., y ter Doest, L. (2003). Negative life events and depressive symptoms in late adolescence: Bonding and cognitive coping as vulnerability factors? Journal of Youth and Adolescence, 32, 185-193.

Lemos, S. (2003). La psicopatología de la infancia y la adolescencia: consideraciones básicas para su estudio. Papeles del Psicólogo, 85, 19-28.

Luthar, S.S. (1991). Vulnerability and resilience: A study of high-risk adolescents. Child Development, 62, 600-616.

Luthar, S.S. (2006). Resilience in development: A synthesis of research across five decades. En D. Cicchetti y D.J. Cohen (Eds.), Developmental Psychopathology: Risk, disorder, and adaptation ( $2^{\mathrm{a}}$ edition, Vol. 3 , pp. 739-795). Nueva York: Wiley.

Luthar, S.S., Cicchetti, D., y Becker, B. (2000). The construct of resilience: A critical evaluation and guidelines for future work. Child Development, 71, 543-562.

Luthar, S.S., y Zelazo, L.B. (2003). Research on resilience: An integrative review. In S.S. Luthar (Ed.), Resilience and vulnerability: Adaptation in the context of childhood adversities (pp. 510-549). Cambridge, RU: Cambridge University Press.

Maggs, J.L., Frome, P.M., Eccles, J.S., y Barber, B.L. (1997). Psychosocial resources, adolescent risk behaviour, and young adult adjustment: Is risk taking more dangerous for some than others? Journal of Adolescence, 20, 103-119.
Manciaux, M., Cyrulnik, B., y Vanistendael. (2003). Résilience. Enfance Majuscule, 72/73, 5-63.

Masten, A. (2001). Ordinary magic: Resilience processes in development. American Psychologist, 56, 227-238.

Masten, A.S., y Wright, M.O.D. (1998). Cumulative risk and protection models of child maltreatment. En B. R. Rossman y M. S. Rosenberg (Eds.), Multiple victimization of children: Conceptual, developmental, research and treatment issues (pp. 7-30). Binghamton, NY: Haworth.

Masten, A.S., Hubbard, J., Gest, S.D., Tellegen, A., Garmezy, N., y Ramírez, M. (1999). Competence in the context of adversity: Pathways to resilience and maladaptation from childhood to adolescence. Development and Psychopathology, 11, 143-169.

Muñoz-Rivas, M.J., y Graña, J.L. (2001). Efectos de riesgo y de protección para el consumo de drogas en adolescentes. Psicothema, 13, 87-94.

McMahon, S.D., Grant, K.E., Compas, B.E., Thurm, A.E., y Ey, S. (2003). Stress and psychopathology in children and adolescents: Is there evidence of specificity? Journal of Child Psychology and Psychiatry and Allied Disciplines: Annual Research Review, 44, 107-133.

Oliva, A. (2006). Relaciones familiares y desarrollo adolescente. Anuario de Psicología, 37, 209-223.

Olson, D.H., Portner, J., y Lavee, Y. (1985). Family Adaptability and Cohesion Scale. University of Minnesota.

Parra. A., y Oliva, A. (2006). Un análisis longitudinal sobre las dimensiones relevantes del estilo parental durante la adolescencia. Infancia y Aprendizaje, 29, 453-470.

Quamma, J.P., y Greenberg, M.T. (1994). Children's experience of life stressors: The role of family social support and social problem-solving skills as protective factors. Journal of Child Clinical Psychology, 23, 295-305.

Stoolmiller, M., y Bank, L. (1995). Autoregressive effects in structural equation models: We see some problems. En J. M. Gottman (Ed.), The Analysis of Change (pp. 261-278). Mahwah, NJ: Lawrence Earlbaum Associates.

Vera, B., Carabelo, B., y Vecina, M.L. (2006). La experiencia traumática desde la psicología positiva: resiliencia y crecimiento postraumático. Papeles del Psicólogo, 27, 40-49. 